\title{
ON SINGULAR SURFACES IN THE DYNAMICS OF CONTINUA WITH MICROSTRUCTURE
}

\author{
BY \\ G. CAPRIZ AND E. G. VIRGA \\ Università di Pisa, Italy
}

1. Introduction. In classical continuum mechanics when a material body $\mathscr{B}$ is traversed by a movable surface $s$, across which a field relevant to the theory suffers discontinuities, a well-established theorem due to Kotchine leads from the integral balance equation for that field in any subbody of $\mathscr{B}$ intersected by $s$ to the balance equation valid on $s$ (cf. Secs. 192 and 193 of [1]). Here we attempt to derive balance equations that should be valid at a surface of discontinuity when the material body can no longer be framed into the dynamical model of classical continuum mechanics, but is rather endowed with a microstructure.

One of the authors of this paper, at least, is of the opinion that a general model for continua with microstructure can be imagined, of which most models of that ilk introduced so far be special cases; he has gone so far as to write a little tract based on that conviction [2].

In his view a privileged observer describes the local microstructure at a place $x$ by assigning it at time $\tau$ a value $\nu(x, \tau)$ chosen among the elements of an appropriate manifold $\mathfrak{m}$ of finite dimension $m$.

On $\mathfrak{m}$ a particular group $\mathscr{G}$ is defined

$$
\mathscr{G}:=\{\mathfrak{g}: \mathfrak{m} \rightarrow \mathfrak{m} \mid \mathfrak{g} \text { is a diffeomorphism }\}
$$

(so that $\mathscr{g}^{\prime}(\nu): \mathscr{T}_{\nu} \rightarrow \mathscr{T}_{\mathscr{g}^{(\nu)}}$, where $\mathscr{T}_{\nu}$ is the tangent space to $\mathfrak{m}$ at $\nu$ ); a differentiable homomorphism $\Gamma$ : $\mathrm{Orth}^{+} \rightarrow \mathscr{G}$ is also available with the whole group $\mathrm{Orth}^{+}$ of the proper orthogonal tensors $Q$ as domain. Correspondingly, the notation

$$
g_{Q}:=\Gamma(Q), \quad \nu_{Q}:=g_{Q}(\nu), \quad Q \in \operatorname{Orth}^{+}
$$

is used. $\Gamma$ and $\mathscr{G}$ are chosen so that an observer placed on a frame rotated at time $\tau$ by $Q(\tau)$ with respect to the privileged frame reads for the microstructure the value $\nu^{*}\left(x^{*}, \tau\right)$ given, in terms of $\nu(x, \tau)$, by

$$
\nu^{*}\left(x^{*}, \tau\right)=\nu(x, \tau)_{Q(\tau)}=g_{Q(\tau)}(\nu(x, \tau)),
$$

where $x^{*}$ is the point corresponding to $x$ in the rotated frame. 
On these geometric premises a general dynamic theory is proposed in [2], a theory which appears to be self-consistent and widely inclusive.

Reviewers of [2] have lamented that the tract is not a treatise and does not give essential space also to one or another missing topic. But one acute reader remarked [12]: "The problem remains of deciding exactly how the new kinematic descriptor should be matched by a force... of deducing the form for new balance equations which are to serve as a basis for equations of evolution of this descriptor... This is the crux of the problem... What is needed is to have a general rule, based on accepted physical principles, which will guide the formulation in each instance. This summum bonum has not yet appeared in the literature, and Capriz cannot supply it here."

This criticism is founded and not easily dismissed: true, special hypotheses abound in the field, but certainly should not be in a tract aiming at essence and generality. Also, the criticism does not admit of an easy answer; the more so because, to some extent, the choice of fundamental axioms is a matter of personal ideas (or preconceptions) of elegance, precedence, and permanence.

We have grounded already in [3] the balance equations proposed in the tract on an appropriate extension of the Nollian theory of interaction among subbodies $b$ of a given body $\mathscr{B}$. One might, however, prefer ground support of a different kind.

For those who admire particularly the compact grace of variational principles, Cosserat's method based on the action euclidienne may be the summum bonum; as the orothers Cosserat write in their famous paper [13, p. 5] "on évitera donc finalement toutes les difficultés et les tâtonnements de la recerche inductive." That method recommends itself particularly in statics; for instance, the vocabulary of most papers on the statics of liquid crystals does not include words such as microstress, equiliberated microforce, etc. There is, however, even here a price to pay.

Some authors assume as primitive an axiom of balance of energy and adjoin to it an axiom of indifference of the formal expression of that equation of balance to changes of observer. The combination of the two assertions has led a number of authors, beginning in 1964 with Green and Rivlin [4] (see also [5] for another early application of this method), to the successful deduction of other equations of balance (of momentum, moment of momentum, micromomentum) as corollaries in many special contexts. The process has been adopted repeatedly in the last thirty years. Recently Gurtin and Podio-Guidugli [10] have proposed to match the invariance principle with a process of scaling from the gross to the fine structure of the body and have thus derived balance equations. All this endeavour may seem to stem from Noll's axiom on frame-indifference of the working of all actions on a body (see [6] and also Sec. I.12 of [7]) and aiming at an explicit rendering of the rôle played by inertial forces, a rôle otherwise left vague. It is our aim to explore here the full power of the method in the general context and to pursue it up to where other techniques fail, i.e., to establish jump conditions at surfaces of discontinuity. To make our path secure, we first exploit the method in Sec. 3 to derive consequences to be interpreted as the basic balance equations for both macromotion and micromotion at regular points. Comparing these equations and those of [2], we may judge the power of the method. We obtain ultimately also the desired jump conditions. 
Special care is obviously required in the choice of the privileged observers with respect to whom the axiom of indifference is posited: to make the right choice is actually of the essence. Such observers are defined in Sec. 2 below. So long as inertia is involved, this issue is rather delicate also in the well-rooted classical domain. In two recent papers, namely [8] and [9], Šilavý deals very neatly with this matter. He envisages in both papers an axiomatic perspective much deeper and more general than ours, a perspective that cannot be easily transferred to our setting.

We adopt throughout the notation of [2] and [3] and, though the paper is selfcontained, we avoid some details and not strictly necessary repetitions.

2. Privileged observers. We begin by completing the remarks of Sec. 1 on general changes of observer. The formulae below are slightly simpler if the vector $q$ of rotation is involved instead of the tensor $\mathbf{Q}$; formally the two quantities are related by

$$
\mathbf{Q}=\mathbf{I}-\mathbf{e} q+\frac{1}{2}(\mathbf{e} q)^{2}-\cdots=e^{-\mathbf{e} q} ;
$$

here $\mathbf{I}$ is the identity tensor and $\mathbf{e}$ the Ricci permutation tensor. Notice that $\mathbf{Q}=$ I if, and only if, $q=0(\bmod 2 \pi u)$, where $u$ is an arbitrary unit vector (this indetermination in $q$ will not worry us since we will be interested only in cases where $|q|$ is small).

An essential rôle is played in our developments by the infinitesimal generator $\mathscr{A}$ of the group action $\mathscr{8}$

$$
\mathscr{A}:=\left.\frac{d g}{d q}\right|_{q=0} ;
$$

$\mathscr{A}$ is assumed to depend smoothly on $\nu$.

To be more specific, the group action $g$ can be represented on a chart of $m$ by

$$
\left(\nu^{*}\right)^{\alpha}=g^{\alpha}(\nu, q) \text { for } \alpha=1, \ldots, m .
$$

The mappings $g^{\alpha}$ satisfy

$$
g^{\alpha}(\nu, 0)=\nu^{\alpha} \text { for } \alpha=1, \ldots, m .
$$

Furthermore, by (2.1) the components of $\mathscr{A}$ are given by

$$
\mathscr{A}_{i}^{\alpha}(\nu):=\left.\frac{d g^{\alpha}}{d q_{i}}\right|_{q=0}
$$

Making use of (2.2), (2.3), and (2.4), we easily evaluate $\nu^{*}, \dot{\nu}^{*}$, and $\ddot{\nu}^{*}$ up to the first order in $|q|,|\dot{q}|$, and $|\ddot{q}|$ :

$$
\begin{gathered}
\left(\nu^{*}\right)^{\alpha}=\nu^{\alpha}+\mathscr{A}_{i}^{\alpha} q_{i}, \\
\left(\dot{\nu}^{*}\right)^{\alpha}=\dot{\nu}^{\alpha}+\frac{\partial \mathscr{A}_{i}^{\alpha}}{\partial \nu^{\beta}} \dot{\nu}^{\beta} q_{i}+\mathscr{A}_{i}^{\alpha} \dot{q}_{i}, \\
\left(\ddot{\nu}^{*}\right)^{\alpha}=\ddot{\nu}^{\alpha}+\frac{\partial \mathscr{A}_{i}^{\alpha}}{\partial \nu^{\beta}} \ddot{\nu}^{\beta} q_{i}+\frac{\partial^{2} \mathscr{A}_{i}^{\alpha}}{\partial \nu^{\beta} \partial \nu^{\gamma}} \dot{\nu}^{\beta} \dot{\nu}^{\gamma} q_{i}+2 \frac{\partial \mathscr{A}_{i}^{\alpha}}{\partial \nu^{\beta}} \dot{\nu}^{\beta} \dot{q}_{i}+\mathscr{A}_{i}^{\alpha} \ddot{q}_{i} .
\end{gathered}
$$


In absolute notation (2.5) reads as follows:

$$
\nu^{*}=\nu+\mathscr{A} q+o(|q|) \text {. }
$$

We learn from (2.6) that, in frames that move one with respect to the other with translational velocity $c$ and rotational velocity $\dot{q}$ but coincide at the instant of observation, two observers read microvelocities $\dot{\nu}$ and $\dot{\nu}^{*}$, respectively, with

$$
\dot{\nu}^{*}=\dot{\nu}+\mathscr{A} \dot{q},
$$

whereas the macrovelocities $\dot{x}^{*}$ and $\dot{x}$ are related by

$$
\dot{x}^{*}=\dot{x}+c+\dot{q} \times\left(x-x^{\prime}\right),
$$

where $x^{\prime}$ is a fixed point.

We say that a motion of $\mathscr{B}$ is rigid if there is an observer for whom both microvelocity and macrovelocity vanish identically on $\mathscr{B}$ (cf. Sec. I.10 of [7] and also Sec. 6 of [3]). Thus, by (2.8) and (2.10), in a rigid motion referred to an arbitrary observer the microvelocity $\dot{\nu}_{R}$ and the macrovelocity $\dot{x}_{R}$ read as follows:

$$
\dot{\nu}_{R}=\mathscr{A} \dot{q}, \quad \dot{x}_{R}=c+\dot{q} \times\left(x-x^{\prime}\right),
$$

where $\mathscr{A}$ is to be evaluated at the value attained by $\nu$ in the motion.

If the rotational velocity $\dot{q}$ vanished identically, but $q$ does not, the macrovelocities are related by

$$
\dot{x}^{*}=\dot{x}+c+q \times \dot{x}+o(|q|) \text {. }
$$

Notice the assumption that translations have no effect on the value of $\nu$.

We now open the way to derive the basic balance equations of continua with microstructure from the balance of energy.

As in classical mechanics, we posit the existence of a privileged observer, who is to write, so to say, these equations. In our developments two special classes of observers, similarly privileged though in different respects, are essential. One class is that of all Galilean observers and the other, ampler class is defined below.

A Galilean change of observer is characterized by

$$
\dot{c} \equiv 0 \text { and } \dot{q} \equiv 0,
$$

whereas neither $c$ nor $q$ need vanish. The effect up to the first order in $|c|$ and $|q|$ of such a change on the macrokinetic energy per unit mass is easily seen (cf. (2.11)) to be

$$
\frac{1}{2}\left(\dot{x}^{*}\right)^{2}=\frac{1}{2} \dot{x}^{2}+\dot{x} \cdot c \text {. }
$$

Likewise, since translations do not affect $\nu$, we require the microkinetic energy per unit mass, a smooth scalar-valued function $\kappa$ of $\nu$ and $\dot{\nu}$, to be invariant under a change of observer that satisfies (2.12). Making use of (2.5) and (2.6), we easily check that such a requirement is satisfied if and only if

$$
\frac{\partial \kappa}{\partial \nu^{\beta}} \mathscr{A}_{i}^{\beta}+\frac{\partial \kappa}{\partial \dot{\nu}^{\beta}} \frac{\partial \mathscr{A}_{i}^{\beta}}{\partial \nu^{\gamma}} \dot{\nu}^{\gamma}=0 \text { for } i=1,2,3 \text {. }
$$

The other class of changes of observer that will be employed below is defined by

$$
\dot{c} \equiv 0 \quad \text { and } \quad \ddot{q} \equiv 0 ;
$$


for simplicity and without loss the observation is referred to a time when $q=0$, as in (2.9) and (2.10).

We record here for later use how both $\kappa$ and $\dot{\kappa}$ are affected up to the first order in $|q|$ by such a change:

$$
\begin{gathered}
\kappa^{*}:=\kappa\left(\nu^{*}, \dot{\nu}^{*}\right)=\kappa+\frac{\partial \kappa}{\partial \dot{\nu}} \cdot \mathscr{A} \dot{q} \\
\left(\kappa^{*}\right)^{\cdot}:=\frac{\partial \kappa}{\partial \nu}\left(\nu^{*}, \dot{\nu}^{*}\right) \cdot \dot{\nu}^{*}+\frac{\partial \kappa}{\partial \dot{\nu}}\left(\nu^{*}, \dot{\nu}^{*}\right) \cdot \ddot{\nu}^{*}=\dot{\kappa}+\left\{\left(\frac{\partial \kappa}{\partial \dot{\nu}}\right)-\frac{\partial \kappa}{\partial \nu}\right\} \cdot \mathscr{A} \dot{q}
\end{gathered}
$$

To arrive at (2.16) some labour is required, based upon use not only of (2.5), (2.6), and (2.7), but also of (2.13). Within the same degree of approximation in both $|c|$ and $|\dot{q}|$ the macrokinetic energy per unit mass transforms as follows:

$$
\frac{1}{2}\left(\dot{x}^{*}\right)^{2}=\frac{1}{2} \dot{x}^{2}+\dot{x} \cdot c+\left(x-x^{\prime}\right) \times \dot{x} \cdot \dot{q} .
$$

3. Balance of energy. The law of balance of energy for any subbody $b$ of $\mathscr{B}$,

$$
\mathscr{E}_{\&}:=\left(\int_{\&} \rho\left(\varepsilon+\frac{1}{2} \dot{x}^{2}+\kappa\right)\right)^{\cdot}-\int_{\&} \rho(f \cdot \dot{x}+\beta \cdot \dot{\nu}+\lambda)-\int_{\partial \mathscr{Q}}(\dot{x} \cdot T n+\dot{\nu} \cdot \mathbf{S} n-h \cdot n)=0
$$

involves, as in the standard case, the density $\varepsilon$ of internal energy, the body force $f$ per unit mass, the rate of (radiant) heating $\lambda$, the Cauchy stress $T$, the heat flux $h$, and the unit vector $n$ of the exterior normal to $\partial b$. But now the microkinetic energy density $\kappa$, the body microforce $\beta$ (an element of the cotangent space $\mathscr{T}_{\nu}^{*}$ ), and the microstress $\mathbf{S}$ also enter the balance law. This law (3.1) is assumed here as a fundamental axiom of the theory we wish to construct.

The second axiom requires (3.1) to apply also when velocity and microvelocity are measured by any privileged observer of the class ruled by (2.14).

Here the different rôle of the two classes of privileged observers defined in Sec. 2 becomes apparent. The invariance of $\kappa$ in the class ruled by (2.12) has allowed us to relate $\kappa$ and $\mathscr{A}$ through (2.13). The invariance of $\mathscr{E}_{\&}$ in the class ruled by (2.14) will lead us from the law of balance of energy to the other balance equations of the theory.

$\mathscr{E}_{\mathcal{Q}}$, as read by any other privileged observer, is

$$
\begin{aligned}
\mathscr{E}_{\ell}^{*}(c, \dot{q}):= & \int_{\mathscr{\sigma}} \rho\left\{\varepsilon+\frac{1}{2}\left(\dot{x}^{*}\right)^{2}+\kappa^{*}\right\} \\
& -\int_{\mathscr{G}} \rho\left\{f \cdot \dot{x}^{*}+\beta \cdot \dot{\nu}^{*}+\lambda\right\}-\int_{\partial \mathscr{C}}\left\{\dot{x}^{*} \cdot T n+\dot{\nu}^{*} \cdot \mathbf{S} n-h \cdot n\right\},
\end{aligned}
$$

where $\dot{x}^{*}$ and $\dot{\nu}^{*}$ are as in (2.10) and (2.9), respectively. In (3.2) use has been made of a classical transport theorem and of the identities

$$
\rho^{*}=\rho, \quad \varepsilon^{*}=\varepsilon, \quad \lambda^{*}=\lambda, \quad f^{*}=f, \quad h^{*}=h, \quad T^{*}=T, \quad n^{*}=n,
$$

which are standard in classical continuum mechanics when $q=0$ (cf., e.g., Sec. I.9 of [7]). In the same spirit, also the following identities have been assumed:

$$
\beta^{*}=\beta, \quad \mathbf{S}^{*}=\mathbf{S} .
$$


We express the invariance of $\mathscr{E}_{\&}$ through the conditions

$$
\left.\frac{\partial \mathscr{E}_{a}^{*}}{\partial c}\right|_{\substack{c=0 \\ \dot{q}=0}}=0,\left.\quad \frac{\partial \mathscr{E}_{a}^{*}}{\partial \dot{q}}\right|_{\substack{c=0 \\ \dot{q}=0}}=0,
$$

intended to be valid identically on the fiber bundle and for all subbodies $b$.

It is a little exercise in elementary analysis (cf. Eqs. (2.16) and (2.17)) to render explicit the two conditions (3.3). When account is also taken of the arbitrariness of the set $b$, the first one leads trivially to the Cauchy equation

$$
\rho \ddot{x}=\rho b+\operatorname{div} T .
$$

The second one is reduced with slightly more bother to read

$$
\mathscr{A}^{\mathrm{T}}\left\{\rho\left(\left(\frac{\partial \kappa}{\partial \dot{\nu}}\right)^{\cdot}-\frac{\partial \kappa}{\partial \nu}\right)-\rho \beta\right\}=\operatorname{div}\left(\mathscr{A}^{\mathrm{T}} \mathbf{S}\right)-\mathbf{e T} .
$$

This equation is equivalent to two conditions, which we proceed now to write. The first condition is that

$$
\operatorname{div}\left(\mathscr{A}^{\mathrm{T}} \mathbf{S}\right)-\mathrm{eT} \in \text { Range of } \mathscr{A}^{\mathrm{T}}
$$

in other words, that there exists an element $\zeta$ in the cotangent space $\mathscr{T}_{\nu}^{*}$ such that

$$
\mathscr{A}^{\mathrm{T}} \zeta=e^{\mathrm{T}}-\left(\operatorname{grad}_{\mathrm{m}} \mathscr{A}^{\mathrm{T}}\right) \mathbf{S}
$$

this relation expresses the local balance of moment of momentum for our body $\mathscr{B}$. It remains for us to require that

$$
\rho\left(\left(\frac{\partial \kappa}{\partial \dot{\nu}}\right)^{\cdot}-\frac{\partial \kappa}{\partial \nu}\right)-\rho \beta+\zeta \in \text { Null Space of } \mathscr{A}^{\mathrm{T}} .
$$

At first sight this requirement seems to be less stringent than the balance of micromomentum in Eq. (8.3) of [2]. Besides, (3.7) involves $\kappa$, instead of $\chi$, the microkinetic co-energy, and the internal actions are valued by the single term $\xi$.

As to the presence of $\kappa$ instead of $\chi$ in (3.7), we recall that $\chi$ appears in (8.3) of [2] to comply with an axiom on microinertia posited in Sec. 7 of [2]. Thus, that axiom is compatible with the invariance of $\mathscr{E}_{\&}$ if $\kappa$ and $\chi$ coincide, which is always the case when $\kappa$ is quadratic with respect to $\dot{\nu}$. Such a feature of $\kappa$ is hereafter understood.

Furthermore, since

$$
(\text { Range of } \mathscr{A})^{\perp}=\text { Null space of } \mathscr{A}^{\mathrm{T}} \text {, }
$$

the right-hand side of (3.7) comprises only the null covector if, and only if,

$$
\text { Range of } \mathscr{A}(\nu)=\mathscr{T}_{\nu} \text { for all } \nu \in \mathfrak{m},
$$

which, in view of (2.9), amounts to saying that the microkinematics of the body is restricted by the condition that all microvelocities be of the type occurring in a rigid 
motion, when $\dot{\nu}$ is in the range of $\mathscr{A} .^{1}$ The class of such continua, though by far not exhaustive (continua with finely distributed voids, for instance, are not included), is quite ample and comprises liquid crystals and Cosserat continua.

We can cite explicitly the case of nematic liquid crystals, a case which can be either described in an intrinsic way, as fits the developments above, or more easily imagined with a prior embedding in ordinary space. We will follow the latter description here. Then $\nu$ (which should be a point belonging to the unit sphere) is thought of as a unit vector $d ; \beta$ and $\zeta$ are also ordinary vectors, $\mathbf{S}$ and $\mathscr{A}$ are second-order tensors. Precisely $\mathscr{A}$ reduces to the tensor ed, so that the range of $\mathscr{A}^{\mathrm{T}}$ comprises all vectors orthogonal to $d$, i.e., the whole of $\mathscr{T}_{\nu}$. Finally the null space of $\mathscr{A}^{\mathrm{T}}$ is the space of all vectors parallel to $d$ (in an intrinsic description, only the null vector of $\mathscr{T}_{\nu}^{*}$ ), so that (3.7) is exactly the equation of balance of micromomentum used in the dynamics of liquid crystals.

We must observe, finally, that the greater stringency of (9.4), (8.3) of [2] in comparison with (3.6), (3.7) above is only apparent. In fact, (3.6) does not determine $\zeta$ uniquely. If $\zeta^{\prime}$ satisfies (3.6), so does $\zeta^{\prime}-\zeta^{\prime \prime}$, provided only $\zeta^{\prime \prime}$ belongs to the null space of $\mathscr{A}^{\mathrm{T}}$. If $\zeta^{\prime}$ satisfies also (3.7), then we can choose

$$
\zeta^{\prime \prime}=\rho\left(\left(\frac{\partial \kappa}{\partial \dot{\nu}}\right)^{\cdot}-\frac{\partial \kappa}{\partial \nu}\right)-\rho \beta+\zeta^{\prime} .
$$

Taking for $\zeta$ the difference $\zeta^{\prime}-\zeta^{\prime \prime}$, we reach the conclusion that $\zeta$ satisfies (3.6) and

$$
\rho\left(\left(\frac{\partial \kappa}{\partial \dot{\nu}}\right)^{\cdot}-\frac{\partial \kappa}{\partial \nu}\right)-\rho \beta+\zeta=0,
$$

which is exactly (8.3) of [2], provided that $\xi$ is substituted by $\xi+\operatorname{div} \mathbf{S}$ and one can give absolute meaning to $\operatorname{div} \mathbf{S}$ (i.e., provided that $\mathfrak{m}$ admits a connection). If this is the case, also (3.6) above reduces precisely to (9.4) of [2].

4. Jump conditions. Here we finally derive the balance equations at a surface of discontinuity.

As is customary, we assume that $s$, the smooth movable surface that traverses the body, is oriented and we denote by $n$ the unit normal and by $u$ the normal speed of displacement of $s$. We further assume that $\nu$ is continuous across $s$, whereas neither $\dot{\nu}$ nor $\dot{x}$ need be. The usual notation for jumps is employed: i.e. $[\cdot]$.

We proceed along the lines of thought followed in Sec. 3, bearing in mind the restrictions to which that method is subject.

We posit a version, fit for our scope, of Kotchine's equation for the balance of energy (the classical Kotchine equation is (241.5) of [1]):

$$
\mathscr{F}:=\left[\rho u\left(\varepsilon+\frac{1}{2} \dot{x}^{2}+\kappa\right)\right]+[\dot{x} \cdot T n+\dot{\nu} \cdot \mathbf{S} n-h \cdot n]=0 .
$$

\footnotetext{
${ }^{1}$ Here we exploit two elementary facts of linear algebra. First, if $\mathscr{L}$ is a linear mapping from a linear space $\mathscr{V}$ into another linear space $\mathscr{W}$, then $\mathscr{L}^{\mathrm{T}}$, the transpose of $\mathscr{L}$, is a linear mapping from $\mathscr{W}^{*}$, the dual of $\mathscr{W}$, into $\mathscr{V}^{*}$, the dual of $\mathscr{V}$. Second, given any subset $\mathscr{U}$ of $\mathscr{V}, \mathscr{U}^{\perp}$ is defined as the subset of $\mathscr{V}^{*}$ consisting of all covectors (i.e., linear forms) that vanish on $\mathscr{U}$ (see, e.g., pp. 71-73 of [11]).
} 
As in Sec. $3 \mathscr{E}_{\&}$ was changed into $\mathscr{E}_{\mathscr{R}}^{*}$ by a change of observer, here $\mathscr{F}$ is changed into

$$
\mathscr{F}^{*}(c, q):=\left[\rho u\left(\varepsilon+\frac{1}{2}\left(\dot{x}^{*}\right)^{2}+\kappa^{*}\right)\right]+\left[\dot{x}^{*} \cdot T n+\dot{\nu}^{*} \cdot \mathrm{S} n-h \cdot n\right],
$$

where again $\dot{x}^{*}$ and $\dot{\nu}^{*}$ are as in (2.10) and (2.9), respectively, and a new identity has been employed, namely

$$
u^{*}=u \text {. }
$$

Paralleling (3.3), we express the invariance of $\mathscr{F}$ under change of observer by requiring that

$$
\left.\frac{\partial \mathscr{F}^{*}}{\partial c}\right|_{\substack{c=0 \\ \dot{q}=0}}=0,\left.\quad \frac{\partial \mathscr{F}^{*}}{\partial \dot{q}}\right|_{\substack{c=0 \\ \dot{q}=0}}=0 .
$$

Inserting (4.2) into (4.3) leads readily to

$$
[\rho u \dot{x}+T n]=0, \quad \mathscr{A}^{\mathrm{T}}\left[\rho u \frac{\partial \kappa}{\partial \dot{\nu}}+\mathbf{S} n\right]=0 .
$$

The former equation is the well-known jump condition for linear momentum of classical mechanics (cf., e.g., (205.3) of [1]), and the latter restricts the jumps of micromomentum.

These equations must be supplemented by the standard kinematical conditions of compatibility (see, e.g., (180.5) of [1]), which for velocity and microvelocity read, respectively, as follows:

$$
\begin{array}{ll}
{[\nabla \dot{x}]=a \otimes n,} & {\left[\frac{\partial \dot{x}}{\partial \tau}\right]=-u a,} \\
{[\nabla \dot{\nu}]=\vartheta \otimes n,} & {\left[\frac{\partial \dot{\nu}}{\partial \tau}\right]=-u \vartheta,}
\end{array}
$$

where $a$ is a vector and $\vartheta$ a co-vector in the appropriate spaces.

When (3.8) applies, (4.4) becomes

$$
\left[\rho u \frac{\partial \kappa}{\partial \dot{\nu}}+\mathbf{S} n\right]=0 .
$$

In particular, for a nematic liquid crystal, taking $\kappa=\frac{1}{2} \dot{d}^{2}$, from (4.5) we get the equation

$$
[\rho u \dot{d}+\mathbf{S} n]=0
$$

whence

$$
[\mathrm{S} n] \cdot d=0,
$$

since $|d|=1$ and so $[d] \cdot d=0$, by the embedding in ordinary space of the manifold relevant for liquid crystals, as described at the close of Sec. 3.

\section{REFERENCES}

[1] C. Truesdell and R. A. Toupin, The classical field theories, Handbuch der Physik, vol. III, Springer, Berlin and New York, 1960

[2] G. Capriz, Continua with microstructure, Springer, Berlin and New York, 1989

[3] G. Capriz and E. G. Virga, Interactions in continua with microstructure, Arch. Rational Mech. Anal. 109, 323-342 (1990) 
[4] A. E. Green and R. S. Rivlin, Simple force and stress multipoles, Arch. Rational Mech. Anal. 16, 325-353 (1964)

[5] A. E. Green, Micro-materials and multipolar continuum mechanics, Internat. J. Engrg. Sci. 3, 533-537 (1965)

[6] W. Noll, La mécanique classique basée sur un axiome d'objectivité, La Méthode Axiomatique dans les Mécaniques Classiques et Nouvelles (Colloq. Internat., Paris, 1959), 47-56, Gauthier-Villars, Paris, 1963

[7] C. Truesdell, $A$ first course in rational continuum mechanics, vol. 1, 2nd ed., Academic Press, New York, 1991

[8] M. Šilhavý, On the concepts of mass and linear momentum in Galilean thermodynamics, Czechoslovak. J. Phys. B 37, 133-157 (1987)

[9] M. Šilhavý, Mass, internal energy, and Cauchy's equations in frame-indifferent thermodynamics, Arch. Rational Mech. Anal. 107, 1-22 (1989)

[10] M. E. Gurtin and P. Podio-Guidugli, On the formulation of mechanical balance laws for structured continua, ZAMP 43, 181-190 (1992)

[11] W. Noll, Finite-dimensional spaces, Nijhoff, Dordrecht, 1987

[12] W. O. Williams, Review of [2], SIAM Rev. 33, 161-163 (1991)

[13] E. Cosserat and F. Cosserat, Théorie des corps déformables, Hermann, Paris, 1909 